\title{
Hemoconcentration is a valuable predictor of prognosis in patients with acute heart failure
}

\author{
QIAN YAN $^{1}$ and SANFENG CHEN ${ }^{2}$ \\ ${ }^{1}$ Department of Emergency, Affiliated Hospital of Hangzhou Normal University; \\ ${ }^{2}$ Department of Internal Medicine, Aged Care Hospital of Zhejiang, Hangzhou, Zhejiang 310015, P.R. China
}

Received March 8, 2019; Accepted September 27, 2019

DOI: $10.3892 /$ etm.2020.8515

\begin{abstract}
The present study was designed to investigate the value of the hemoconcentration (HCT) in predicting the prognosis of patients with acute heart failure (AHF). A total of 188 patients with AHF were enrolled in the present retrospective study and divided into four groups based on their HCT values. The endpoint was either cardiac-associated death or re-hospitalization due to aggravated HF. The 2-year survival rates of patients in these four groups were compared. The area under the receiver operating characteristic curve (AUC) was determined to evaluate the significance of HCT for assessing the prognosis of patients with AHF. Cox-proportional hazards regression models were performed to determine whether the HCT is an independent factor for predicting the prognosis of patients with AHF in comparison with other traditional predictors, including B-type natriuretic peptide (BNP) and creatinine. Of these 188 patients with AHF, 99 experienced aggravated cardiac HF resulting in death or re-hospitalization within 2 years. The AUC for HCT, as a prognostic criterion, was 0.610 (95\% confidence interval: $0.528-0.691, \mathrm{P}<0.001)$ with a sensitivity of $54.5 \%$ and a specificity of $65.2 \%$. Kaplan-Meier analysis indicated that patients with a higher HCT had a lower rate of death or re-hospitalization due to cardiogenic events $\left(\chi^{2}=9.442, \mathrm{P}=0.024\right)$. Cox regression analysis revealed that HCT, hemoglobin, BNP, New York Heart Association cardiac function classification and serum creatinine were independent prognostic factors in AHF. HCT may serve as a valuable predictor of prognosis in patients with AHF. Compared with
\end{abstract}

Correspondence to: Dr Sanfeng Chen, Department of Internal Medicine, Aged Care Hospital of Zhejiang, 21 Jinhua Road, Hangzhou, Zhejiang 310015, P.R. China

E-mail: hsdfyxnk@163.com

Abbreviations: AHF, acute heart failure; BNP, B-type natriuretic peptide; NYHA, New York Heart Association; BMI, body mass index; LVEF, left ventricular ejection fraction; LVMI, left ventricular mass index; LVEDD, left ventricular end diastolic diameter

Key words: acute heart failure, hemoconcentration, B-type natriuretic peptide that of BNP, measurement of the HCT is more convenient and economical and may be widely performed at primary hospitals.

\section{Introduction}

Heart failure (HF) may be chronic, i.e., develop over a long duration, or acute, i.e., with a rapid onset of clinical manifestations (1). While chronic HF (CHF) is usually the end stage of a variety of cardiovascular diseases, acute $\mathrm{HF}$ (AHF) may be caused by numerous factors that exacerbate $\mathrm{CHF}$ in a short period or result in a sudden heart attack (2). AHF, which most commonly involves acute left ventricular failure, is potentially life-threatening and requires immediate in-hospital treatment. Even so, the in-hospital mortality rate and readmission rate after discharge are relatively high (3). Several cardiac functional indexes, including left ventricular size and ejection fraction (EF), and certain cardiac functional classification methods, including the New York Heart Association (NYHA) classification, have been used to assess the severity and outcomes of AHF, but each of them has certain limitations $(4,5)$. Furthermore, several biomarkers, including serum B-type natriuretic peptide (BNP) and creatinine, have been identified and widely used to assess the severity of AHF and predict the prognosis of patients with AHF (6). However, the circulating levels of BNP may be affected by certain other diseases, including valvular heart disease (7), while the serum creatinine concentration is more associated with kidney dysfunction (6). Thus, it remains imperative to identify novel biomarkers for AHF that are reliable and specific.

Recently, the hemoconcentration (HCT), an index reflecting a rapid increase in the concentration of red blood cells in the blood, has received attention from clinicians. An elevated HCT has been reported to be associated with more severe damage to the brain in patients with hemolytic uremic syndrome (8). Correlations between HCT and heart diseases have been explored, but conflicting results have been reported. For instance, HCT was indicated to be closely associated with a more extensive weight loss and elevated risk of exacerbated kidney function in patients with AHF during hospitalization (9), whereas the data from the Korean Heart Failure Registry suggested that increased HCT may be beneficial for patients with AHF without hyponatremia (10). However, the ANCHOR study indicated that either high or low HCT predicted worse outcomes in HF patients, including 
death and re-hospitalization (11). The PRAISE study indicated that anemia, in which the HCT is decreased, is an independent risk factor for patients with severe HF (12). Hence, the effectiveness of HCT to predict the prognosis of AHF patients remains elusive.

In the present study, the associations of different levels of HCT with the outcome of AHF were investigated, and the prognostic value of HCT for patients with AHF was examined and compared with that of other well-recognized traditional biomarkers for AHF, including BNP and serum creatinine. The aim of the present study was to clarify whether HCT may serve as a prognostic biomarker in patients with AHF. The results suggest that HCT may be applied as a valuable prognostic factor for patients with AHF.

\section{Materials and methods}

Ethics statement. The protocol of the present study was approved by the Ethics Committee of the Affiliated Hospital of Hangzhou Normal University (Hangzhou, China). All patients who participated in the study were informed of the purpose of the study and provided written informed consent.

Patient selection. 523 consecutive patients diagnosed with any AHF at the Department of Critical Care Medicine, Affiliated Hospital of Hangzhou Normal University (Hangzhou, China) between June 2013 and June 2015 were selected for the present retrospective study. All cases met the diagnostic criteria for AHF based on the Guidelines for the Diagnosis and Treatment of Acute Heart Failure of the American Heart Association from 2010 (13). Patients fulfilling all of the following criteria were included in the present study: i) Diagnosis of AHF established based on medical history, associated cause(s), clinical manifestations and echocardiographic findings; ii) NYHA classification grade III to IV; iii) systolic/diastolic blood pressure of $\geq 90 / 60 \mathrm{mmHg}(1 \mathrm{mmHg}=133 \mathrm{~Pa})$; and iv $)$ age between 45 and 85 years. Patients with one of the following were excluded from the present study: i) Severe valvular stenosis, constrictive pericarditis, restrictive or hypertrophic cardiomyopathy; ii) severe pulmonary hypertension, severe ventricular arrhythmia, cardiogenic shock or insufficient blood volume; iii) severe hypotension, malignant tumors, trauma or infection; or iv) severe liver or kidney dysfunction. After application of the inclusion and exclusion criteria, a total of 188 patients were finally included during the 2-year follow-up period of the present study.

Data collection. The demographic and baseline clinical characteristics of all patients were collected from the electronic medical record system of the hospital that had been entered for each patient on admission, including age, gender, body mass index (BMI), current diseases, comorbidities, past medical history, medication status and cardiac function according to the NYHA cardiac function classification.

End-points. The end-point event of the present study was either cardiac-associated death or re-hospitalization due to AHF. All patients were followed up for 2 years or until the end-point occurred. According to the serum HCT values on admission, the patients were divided into four groups as follows: $\geq 40 \%$ group, 36-39\% group, 30-35\% group and $<30 \%$ group. The incidence of end-point events during the 2-year follow-up period was compared among these four groups.

Blood tests. Blood samples were collected from each patient on admission and the HCT, as well as the levels of BNP, hemoglobin, creatinine, uric acid, low-density lipoprotein (LDL) and total cholesterol (TC) were measured.

Echocardiography. Echocardiography was performed using an EPIQ 7 ultrasound machine (Philips) for all participants and cardiac function indexes, including left ventricular EF (LVEF), left ventricular mass index (LVMI) and left ventricular end diastolic diameter (LVEDD) were determined.

Statistical analysis. All statistical analyses were performed by SPSS 19.0 software (IBM, Corp.). Continuous variables, which are normally distributed, were expressed as the mean and standard deviation, comparisons of these data between groups were performed by a student's t-test. In addition, median and interquartile ranges were analyzed when the variables were non-normally distributed, such as in the case of BNP. Correlations between the two sets were performed using Spearman's correlation analysis. Categorical variables were expressed as count and percentage, and were analyzed by the chi-square test. Kaplan-Meier analysis was used to evaluate survival curve, which were compared using a log-rank test. Cox proportional hazard models were used to determine the risk factors influencing the prognosis, including cardiac-associated death or re-hospitalization due to AHF. The area under the receiver operating characteristic (ROC) curve (AUC) was assessed to determine the ability of factors to predict the prognosis of AHF patients. $\mathrm{P}<0.05$ was considered to indicate statistical significance.

\section{Results}

Demographic and basic clinical characteristics. A total of 188 patients [mean age, $54.8( \pm 11.3)$ years; 110 males and 78 females] were enrolled in the present study during the 2-year follow-up period. Of these, 99 patients experienced either cardiac-associated death or re-hospitalized due to AHF within the study period, corresponding to a rate of $52.7 \%$. The average time from first hospitalization to the occurrence of the end-point event was 10.5 months.

Comparison of clinical data among patients in different HCT groups. The 188 patients were divided into four groups based on the HCT values: The $\geq 40 \%$ group $(n=51), 36-39 \%$ group $(n=46), 30-35 \%$ group $(n=56)$ and the $<30 \%$ group $(n=35)$. The clinical data were compared among these different HCT groups. As presented in Table I, there were significant differences among these groups in the BNP level, hemoglobin level, creatinine concentration, NYHA grade, and 1-year and 2-year survival rates $(\mathrm{P}<0.05)$, but no differences were present with regard to age, gender, comorbidities and BMI. However, there were no significant differences in the proportion of patients treated with angiotensin-converting enzyme inhibitor vs. angiotensin receptor blocker, or in the serum lipid levels, uric acid concentration, LVEF, LVMI or LVEDD among these 
Table I. Comparison of clinical data of patients of different HCT groups.

\begin{tabular}{|c|c|c|c|c|c|}
\hline \multirow[b]{2}{*}{ Item } & \multicolumn{4}{|c|}{$\mathrm{HCT}(\%)$} & \multirow[b]{2}{*}{ P-value } \\
\hline & $\geq 40(\mathrm{n}=51)$ & $36-39(n=46)$ & $30-35(n=56)$ & $<30(\mathrm{n}=35)$ & \\
\hline Age (years) & $56.8 \pm 9.3$ & $55.4 \pm 10.2$ & $53.6 \pm 11.7$ & $53.4 \pm 14.2$ & 0.893 \\
\hline Sex (male/female) & $26 / 25$ & $28 / 18$ & $35 / 21$ & $21 / 14$ & 0.638 \\
\hline Diabetes (yes/no) & $11 / 40$ & $10 / 36$ & $10 / 46$ & $6 / 29$ & 0.919 \\
\hline ACEI/ARB (yes/no) & $17 / 34$ & $14 / 32$ & $18 / 38$ & $12 / 23$ & 0.984 \\
\hline BMI $\left(\mathrm{kg} / \mathrm{m}^{2}\right)$ & $22.9 \pm 5.4$ & $22.3 \pm 3.8$ & $23.1 \pm 4.1$ & $23.7 \pm 3.5$ & 0.934 \\
\hline Hemoglobin $(\mathrm{g} / \mathrm{l})$ & $121.8 \pm 12.5$ & $116.8 \pm 13.4$ & $110.8 \pm 12.4$ & $104.8 \pm 16.9$ & 0.032 \\
\hline $\mathrm{BNP}(\mathrm{pg} / \mathrm{ml})$ & $2,470(1,249-4,535)$ & $2,684(1,361-4,883)$ & $3,125(1,530-5,187)$ & $3,470(1,919-6,735)$ & 0.015 \\
\hline Creatinine $(\mu \mathrm{mol} / \mathrm{l})$ & $105.4 \pm 24.5$ & $113.8 \pm 21.6$ & $118.6 \pm 30.2$ & $123.9 \pm 35.7$ & 0.026 \\
\hline Uric acid $(\mu \mathrm{mol} / \mathrm{l})$ & $324.5 \pm 56.9$ & $346.2 \pm 67.5$ & $339.4 \pm 44.5$ & $347.1 \pm 58.2$ & 0.763 \\
\hline $\mathrm{LDL}(\mathrm{mmol} / \mathrm{l})$ & $3.25 \pm 0.47$ & $3.41 \pm 0.75$ & $3.47 \pm 0.83$ & $3.38 \pm 0.91$ & 0.682 \\
\hline $\mathrm{TC}(\mathrm{mmol} / \mathrm{l})$ & $5.37 \pm 1.27$ & $5.61 \pm 1.62$ & $5.17 \pm 0.54$ & $5.38 \pm 1.31$ & 0.863 \\
\hline NYHA classification (II/III/IV) & $32 / 14 / 5$ & $23 / 15 / 8$ & $30 / 17 / 9$ & $8 / 16 / 11$ & $<0.001$ \\
\hline $\mathrm{EF}(\%)$ & $39.3 \pm 7.2$ & $35.6 \pm 10.1$ & $32.4 \pm 8.4$ & $29.9 \pm 10.6$ & 0.641 \\
\hline LVMI (mm) & $151.7 \pm 18.2$ & $153.6 \pm 23.4$ & $154.3 \pm 19.9$ & $153.7 \pm 20.5$ & 0.932 \\
\hline $\operatorname{LVEDD}(\mathrm{mm})$ & $65.1 \pm 9.4$ & $65.2 \pm 11.3$ & $66.2 \pm 9.2$ & $67.0 \pm 11.9$ & 0.857 \\
\hline 1-year survival rate $(\%)$ & 74.5 & 71.7 & 66.0 & 45.7 & 0.033 \\
\hline 2-year survival rate $(\%)$ & 60.8 & 52.2 & 41.1 & 28.6 & 0.036 \\
\hline
\end{tabular}

Unless otherwise specified, values are expressed as the mean \pm standard deviation, $\mathrm{n}$ or median (interquartile range). HCT, hemoconcentration; LDL, low-density lipoprotein; TC, total cholesterol; EF, ejection fraction; LVMI, left ventricular mass index; LVEDD, left ventricular end diastolic diameter; BMI, body mass index; BNP, B-type natriuretic peptide; ACEI, angiotensin-converting enzyme inhibitor; ARB, angiotensin receptor blocker; NYHA, New York Heart Association.

groups ( $\mathrm{P}>0.05$; Table I). In addition, Spearman correlation analysis suggested that HCT was negatively correlated with $\log \mathrm{BNP}(\mathrm{r}=-0.629, \mathrm{P}=0.024$; Fig. 1).

Comparison of cardiac event-free rates among different HCT groups. Kaplan-Meier analysis was performed to compare the cumulative survival rates of patients among the four HCT groups. The log-rank test result was $\chi^{2}=9.442$ with $\mathrm{P}=0.024$ and the cumulative survival rate was significantly higher in the higher HCT groups than in the lower HCT group (Fig. 2).

Predictive value of HCT regarding cardiac event-free of patients. A ROC curve analysis was performed to evaluate the predictive value of the $\mathrm{HCT}$ regarding the survival outcome for patients with AHF. As presented in Fig. 3, the ROC curve analysis indicated that serum HCT values were predictive of survival of patients with AHF, with an AUC of 0.610 [95\% confidence interval (CI): 0.528-0.691, $\mathrm{P}<0.001$ ], at cutoff point of HCT is $35.45 \%$, the sensitivity and specificity were 54.5 and $65.2 \%$, respectively (Fig. 3 ).

Determination of the association between HCT and recurrent cardiac events or re-hospitalization due to AHF. Cox proportion hazards regression analysis was performed to determine the influence of various factors, including the HCT, BNP, serum creatinine and NYHA classification, on the recurrent cardiac or re-hospitalization due to AHF. Demographic variables including age, sex and $\mathrm{BMI}$, and clinical variables

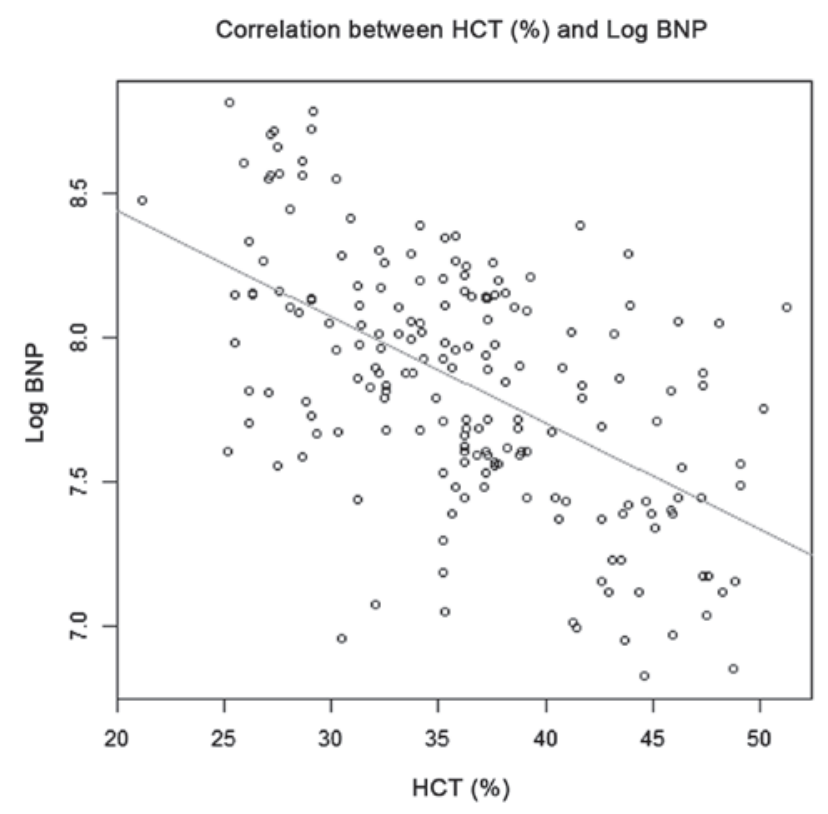

Figure 1. Correlation between HCT (\%) and Log BNP in patients with acute heart failure.

including HCT, ACEI/ARB, hemoglobin, BNP, serum creatinine, uric acid, LDL, TC, NYHA classification, and diabetes were analyzed using the Cox proportional hazards model. The results revealed that HCT, sex, ACEI/ARB, hemoglobin, 


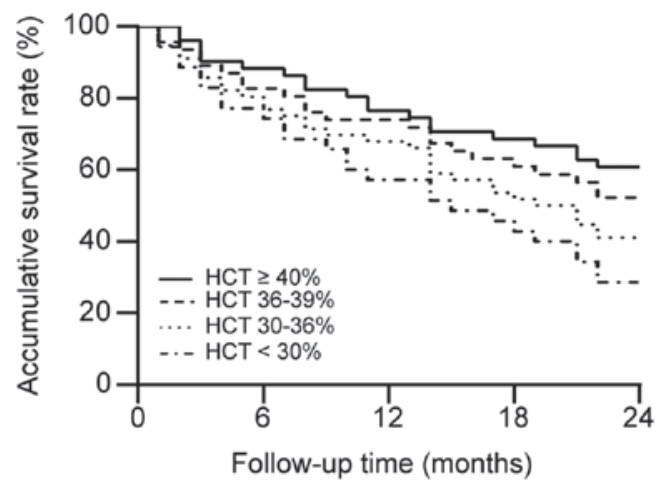

Figure 2. Comparison of survival rates among patients in different HCT groups. HCT, hemoconcentration.

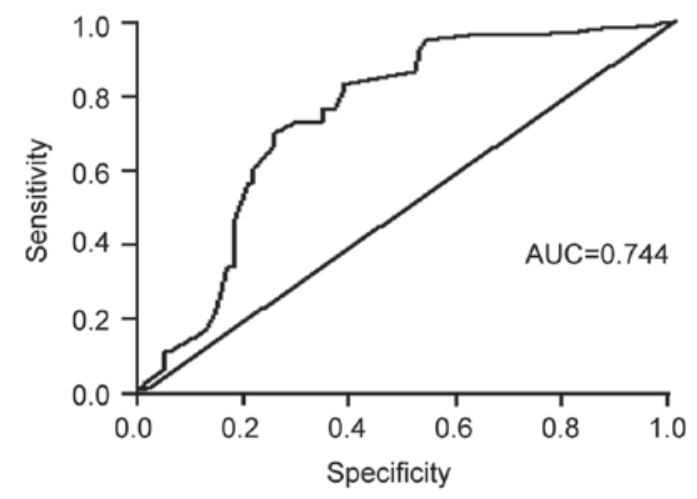

Figure 3. Determination of the association between hemoconcentration and outcomes in patients with acute heart failure. AUC, area under the receiver operating characteristics curve. Cutoff point, $35.45 \%$ of hemoconcentration.

$\log$ of BNP, serum creatinine and NUHA classification were significantly associated with recurrent cardiac events (Table II; Model 1). Males [hazard ratio (HR), 0.08; $\mathrm{P}<0.001$; reference, female], with ACEI/ARB (HR, 0.01; $\mathrm{P}<0.001$; reference, without ACEI/ARB), higher HCT (HR, 0.96; $\mathrm{P}=0.019)$, higher hemoglobin (HR, 0.98; $\mathrm{P}=0.009)$, lower BNP (log BNP's HR, 2.38; $\mathrm{P}<0.001)$, lower serum creatinine $(\mathrm{HR}, 1.01 ; \mathrm{P}=0.003)$ and lower classes of NYHA [HR (class III), 26.82; $\mathrm{P}<0.001$; HR (class IV), 98.10; $\mathrm{P}<0.001$; reference, class II] had a lower risk of cardiac event recurrence (Table II; Model 1).

After adjustment for sex, ACEI/ARB, hemoglobin, Log of BNP, serum creatinine, uric acid, LDL, TC, NYHA classification and diabetes, it revealed that a higher HCT value was associated with a lower risk of recurrent cardiac events (HR, 0.48; P=0.098; Table II; Model 2).

\section{Discussion}

Prior research has indicated that HCT is closely linked to the health status of patients with CHF. For instance, a previous study reported a significant correlation between increased levels of HCT and a better quality of life in patients with AHF (14), and other studies suggested that a decreased HCT and impaired kidney function are risk factors for mortality in patients with CHF (11). However, the association of HCT with the short-term outcomes of acute decompensated HF has remained largely elusive. In the present study, the association of
HCT with the outcomes of AHF patients, i.e., cardiogenic death or re-hospitalization, were examined, and a higher HCT was indicated to be linked to a reduced risk of cardiac-associated events in patients with AHF. The present results also suggest that, similar to BNP and serum creatinine, HCT may serve as an independent prognostic factor for patients with AHF.

$\mathrm{HF}$ is a common condition and a leading cause of hospitalization worldwide (15). Compared with CHF, AHF is associated with higher cardiogenic mortality, a higher re-hospitalization rate and more cardiovascular events due to the rapid decrease in myocardial contractility and increase in cardiac load, resulting in a sudden drop in acute cardiac output, increased pulmonary circulation pressure and elevated peripheral circulation resistance (16). Due to weakened left ventricular systolic function accompanied by a decreased glomerular filtration rate and increased reabsorption of water and sodium by renal tubules, the blood volume is frequently increased, subsequently resulting in sodium retention and dilute anemia, as well as decreased hemoglobin and HCT (17). Thus, HCT is an indicator reflecting the severity of anemia and sodium retention, which are linked to kidney dysfunction and affect the prognosis of patients with HF (18). HCT has therefore been considered as another predictor of the prognosis of patients with AHF. Indeed, the results of the present study support this notion: i) In patients with decompensated AHF, a higher HCT value on admission was significantly associated with a higher survival rate during the 2-year follow-up period compared with a lower HCT value, ii) ROC curve analysis indicated that the sensitivity and specificity of HCT for predicting cardiac events were 83.3 and $63.7 \%$, respectively, and iii) multivariate Cox analysis suggested that the HCT value on admission is an independent predictor of mortality of AHF patients. These observations were consistent with those of a previous study indicating that HCT is a predictor of short-term prognosis in AHF patients after adjustment for risk factors for traditional cardiogenic events (19). Thus, it may be concluded that patients with AHF with a higher HCT have a lower risk of any recurrence, which are in turn linked to cardiac-associated death and re-hospitalization, and this association is independent of traditional risk factors, including Log BNP, NYHA classification and serum creatinine. While the exact mechanisms underlying the association between HCT and the prognosis of patients with AHF remain elusive, multiple factors are likely to be implicated. For instance, the association between HCT and kidney function may be involved (20).

Of note, conflicting results were reported regarding the association between HCT and the outcomes for patients with HF. For instance, the ANCHOR study suggested that HCT, either high or low, was predictive of unfavorable outcomes in patients with HF (11), but a Korean study supported a beneficial role of HCT in non-hyponatremic patients with AHF (10). In addition, the PRAISE study revealed an association between a decreased HCT and the severity of HF (12). The exact reasons for these discrepancies remain elusive, but they may, at least partially, be attributed to the fact that the patients selected in those studies had different comorbidities. The results of the present study support an association between high HCT and favorable outcomes in AHF patients.

BNP and serum creatinine are well-recognized prognostic biomarkers for patients with HF. Regarding the production of 
Table II. Analysis of the factors affecting the recurrent cardiac events of patients with acute heart failure.

\begin{tabular}{|c|c|c|c|c|}
\hline \multirow[b]{2}{*}{ Variable } & \multicolumn{2}{|c|}{ Model $1^{\mathrm{a}}$} & \multicolumn{2}{|l|}{ Model $2^{\mathrm{b}}$} \\
\hline & Crude HR (95\% CI) & $\mathrm{P}$-value & Adjusted HR (95\% CI) & $\mathrm{P}$-value \\
\hline Age & $0.99(0.98-1.01)$ & 0.549 & - & - \\
\hline \multicolumn{5}{|l|}{ Sex } \\
\hline Male vs. female & $0.08(0.05-014)$ & $<0.001$ & $0.35(0.18-0.67)$ & 0.002 \\
\hline \multicolumn{5}{|l|}{ ACEI/ARB } \\
\hline Yes vs. no & $0.01(0.001-0.08)$ & $<0.001$ & $0.54(0.01-0.43)$ & 0.006 \\
\hline BMI $\left(\mathrm{kg} / \mathrm{m}^{2}\right)$ & $0.97(0.92-1.02)$ & 0.266 & - & - \\
\hline $\operatorname{HCT}(\%)$ & $0.96(0.93-0.99)$ & 0.019 & $0.48(0.20-1.14)$ & 0.098 \\
\hline Hemoglobin (g/l) & $0.98(0.97-0.99)$ & 0.009 & $1.02(0.92-1.12)$ & 0.737 \\
\hline $\log$ BNP (pg/ml) & $2.38(1.50-3.77)$ & $<0.001$ & $0.17(0.006-4.93)$ & 0.302 \\
\hline Serum creatinine $(\mu \mathrm{mol} / \mathrm{l})$ & $1.01(1.00-1.02)$ & 0.003 & $0.90(0.94-1.04)$ & 0.685 \\
\hline Uric acid $(\mu \mathrm{mol} / \mathrm{l})$ & $1.00(0.99-1.00)$ & 0.845 & $1.00(0.99-1.01)$ & 0.07 \\
\hline $\mathrm{LDL}(\mathrm{mmol} / \mathrm{l})$ & $0.85(0.65-1.11)$ & 0.239 & $0.83(0.60-1.13)$ & 0.238 \\
\hline TC (mumol/l) & $1.03(0.88-1.21)$ & 0.697 & $0.92(0.76-1.12)$ & 0.422 \\
\hline \multicolumn{5}{|c|}{ NYHA classification (II/III/IV) } \\
\hline II vs. III & $26.82(12.45-57.78)$ & $<0.001$ & $0.01(0.00-9.29)$ & 0.191 \\
\hline III vs. IV & $98.10(42.85-224.59)$ & $<0.001$ & $0.02(0.00-23.39)$ & 0.284 \\
\hline \multicolumn{5}{|l|}{ Diabetes } \\
\hline No vs. yes & $0.00(0.00-$ infc $)$ & 0.994 & $0.00\left(0.00-\right.$ inf $\left.^{c}\right)$ & 0.996 \\
\hline LVMI (g/m²) & $1.00(0.99-1.01)$ & 0.659 & - & - \\
\hline LVEDD (mm) & $1.00(0.98-1.02)$ & 0.823 & - & - \\
\hline
\end{tabular}

${ }^{a}$ Univariate and ${ }^{b}$ multivariate analysis were performed. ${ }^{\mathrm{c}} \mathrm{Model}$ is not converged. HZ, hazard ratio; CI, confidence interval; ACEI, angiotensin-converting enzyme inhibitor; ARB, angiotensin receptor blocker; BMI, body mass index; BNP, B-type natriuretic peptide; HCT, hemoconcentration; LDL, low-density lipoprotein; TC, total cholesterol; NYHA, New York Heart Association; LVMI, left ventricular mass index; LVEDD, left ventricular end diastolic diameter.

BNP, a precursor, NT-pro-BNP, is synthesized and secreted mainly by the ventricular myocytes, and it exhibits a variety of biological activities, including diuresis, suppression of $\mathrm{Na}^{+}$transport, dilation of blood vessels and inhibition of the renin-angiotensin-aldosterone system (21). In a number of heart diseases, including acute myocardial infarction, congenital heart disease and heart failure, an elevated concentration of BNP in the blood stream has been observed (22-24). A meta-analysis of 40 prospective studies revealed that elevated circulating levels of BNP were closely associated with an increased risk of cardiovascular disease (25), and another study suggested that BNP, but not left ventricular cardiac function, held a strong prognostic value regarding outcomes for patients with HF (26). Consistent with the above results, the present study indicated that BNP was an independent risk factor for poor outcome in patients with AHF. It was also revealed that HCT, as another independent prognostic factor in $\mathrm{AHF}$ patients, exhibited a significant negative correlation with the Log BNP. Collectively, these observations suggest that BNP and HCT may serve as prognostic predictors for outcomes in patients with HF. However, compared with the measurement of BNP, the detection of HCT is more convenient, cost-efficient and technologically reliable. At present, HCT is one of the widely used clinical indicators in a number of diseases. Hence, determination of HCT in patients with AHF should be more acceptable for patients and their families and may be widely performed at primary hospitals. Serum creatinine is linked to the glomerular filtration rate and mainly reflects the kidney function, and high levels of serum creatinine may indicate the severity of HF (27). In the present study, all three factors, BNP, serum creatinine and HCT, were determined to be independent predictors of the prognosis of patients with AHF. It has been reported that BNP and serum creatinine are valuable factors for risk stratification of patients with HF (28-30). In the future, it may be worthwhile to explore whether inclusion of BNP, serum creatinine and HCT into risk assessment models together with other traditional cardiovascular risk factors increases the accuracy of disease discrimination.

The limitations of the present study should be pointed out. First, the study cohort was small and due to the retrospective nature of the study, it was not possible to determine any cause-effect associations. Large-cohort multi-center prospective studies are required in order to corroborate the present results and conclusions. Furthermore, the follow-up time was 2 years, which was relatively short, and it has been well documented that the incidence of cardiac events in patients with AHF increases significantly over time. Therefore, dynamic changes in HCT and their correlation with the long-term 
outcomes for patients with AHF warrant further exploration in the future. In addition, the present study focused on the association of HCT with acute heart failure, and thus, troponin levels and HCT were not compared in the present study. However, troponin levels will be included in future studies by our group and there are plans to expand the present study to further corroborate the major findings from the present results.

In conclusion, patients with AHF with a low HCT have a higher risk of recurrent cardiac events and the HCT is an effective predictor of outcomes for patients with AHF.

\section{Acknowledgements}

Not applicable.

\section{Funding}

No funding was received.

\section{Availability of data and materials}

The datasets used and/or analyzed during the present study are available from the corresponding author on reasonable request.

\section{Authors' contributions}

QY analyzed and interpreted the patient data regarding the hematological disease and the transplant. SFC performed the histological examinations of the kidneys, and was a major contributor in writing the manuscript. All authors read and approved the final manuscript.

\section{Ethics approval and consent to participate}

The study protocol was approved by the Ethics Committee of Hangzhou Normal University Affiliated Hospital. All patients who participated in the study were aware of the purpose of the study and provided written informed consent.

\section{Patients' consent for publication}

Not applicable.

\section{Competing interests}

The authors declare that they have no competing interests.

\section{References}

1. Gheorghiade M, Zannad F, Sopko G, Klein L, Piña IL, Konstam MA, Massie BM, Roland E, Targum S, Collins SP, et al: Acute heart failure syndromes: Current state and framework for future research. Circulation 112: 3958-3968, 2005.

2. Kurmani S and Squire I: Acute heart failure: Definition, classification and epidemiology. Current Heart Fail Rep 14: 385-392, 2017.

3. Felker GM, Lee KL, Bull DA, Redfield MM, Stevenson LW, Goldsmith SR, LeWinter MM, Deswal A, Rouleau JL, Ofili EO, et al: Diuretic strategies in patients with acute decompensated heart failure. N Engl J Med 364: 797-805, 2011.

4. Fonarow GC: Refining classification of heart failure based on ejection fraction. JACC Heart Fail 5: 808-809, 2017.
5. Raphael C, Briscoe C, Davies J, Ian Whinnett Z, Manisty C, Sutton R, Mayet J and Francis DP: Limitations of the New York Heart Association functional classification system and self-reported walking distances in chronic heart failure. Heart 93: 476-482, 2007.

6. Richards AM: Biomarkers in acute heart failure-cardiac and kidney. Card Fail Rev 1: 107-111, 2015.

7. Ray SG: Natriuretic peptides in heart valve disease. Heart 92 : 1194-1197, 2006

8. Ardissino G, Daccò V, Testa S, Civitillo CF, Tel F, Possenti I, Belingheri M, Castorina P, Bolsa-Ghiringhelli N, Tedeschi S, et al: Hemoconcentration: A major risk factor for neurological involvement in hemolytic uremic syndrome. Pediatr Nephrol 30: 345-352, 2015.

9. Davila C, Reyentovich A and Katz SD: Clinical correlates of hemoconcentration during hospitalization for acute decompensated heart failure. J Card Fail 17: 1018-1022, 2011.

10. Oh J, Kang SM, Kim IC, Han S, Yoo BS, Choi DJ, Kim JJ, Jeon ES, Cho MC, Oh BH, et al: The beneficial prognostic value of hemoconcentration is negatively affected by hyponatremia in acute decompensated heart failure: Data from the Korean Heart Failure (KorHF) Registry. J Cardiol 69: 790-796, 2017.

11. Go AS, Yang J, Ackerson LM, Lepper K, Robbins S, Massie BM and Shlipak MG: Hemoglobin level, chronic kidney disease, and the risks of death and hospitalization in adults with chronic heart failure: The Anemia in Chronic Heart Failure: Outcomes and Resource Utilization (ANCHOR) Study. Circulation 113: 2713-2723, 2006.

12. Mozaffarian D, Nye R and Levy WC: Anemia predicts mortality in severe heart failure: The prospective randomized amlodipine survival evaluation (PRAISE). J Am Coll Cardiol 41: 1933-1939, 2003.

13. Lloyd-Jones D, Adams RJ, Brown TM, Carnethon M, Dai S, De Simone G, Ferguson TB, Ford E, Furie K, Gillespie C, et al: Executive summary: Heart disease and stroke statistics-2010 update: A report from the American Heart Association. Circulation 121: 948-954, 2010.

14. Testani JM, Brisco MA, Chen J, McCauley BD, Parikh CR and Tang WH: Timing of hemoconcentration during treatment of acute decompensated heart failure and subsequent survival: Importance of sustained decongestion. J Am Coll Cardiol 62: 516-524, 2013.

15. O'Connell JB and Bristow MR: Economic impact of heart failure in the United States: Time for a different approach. J Heart Lung Transplant 13: S107-S112, 1994.

16. Yancy CW, Lopatin M, Stevenson LW, De Marco T and Fonarow GC; ADHERE Scientific Advisory Committee and Investigators: Clinical presentation, management, and in-hospital outcomes of patients admitted with acute decompensated heart failure with preserved systolic function: A report from the Acute Decompensated Heart Failure National Registry (ADHERE) Database. J Am Coll Cardiol 47: 76-84, 2006.

17. Waldum B, Westheim AS, Sandvik L, Flønæs B, Grundtvig M, Gullestad L, Hole T and Os I: Baseline anemia is not a predictor of all-cause mortality in outpatients with advanced heart failure or severe renal dysfunction. Results from the Norwegian Heart Failure Registry. J Am Coll Cardiol 59: 371-378, 2012.

18. Alexandrakis MG and Tsirakis G: Anemia in heart failure patients. ISRN Hematol 2012: 246915, 2012.

19. Oh J, Kang SM, Hong N, Youn JC, Han S, Jeon ES, Cho MC, Kim JJ, Yoo BS, Chae SC, et al: Hemoconcentration is a good prognostic predictor for clinical outcomes in acute heart failure: Data from the Korean Heart Failure (KorHF) Registry. Int J Cardiol 168: 4739-4743, 2013.

20. Ter Maaten JM, Valente MA, Damman K, Cleland JG, Givertz MM, Metra M, O'Connor CM, Teerlink JR, Ponikowski P, Bloomfield DM, et al: Combining diuretic response and hemoconcentration to predict rehospitalization after admission for acute heart failure. Circ Heart Fail 9, 2016.

21. Cataliotti A, Boerrigter G, Costello-Boerrigter LC, Schirger JA, Tsuruda T, Heublein DM, Chen HH, Malatino LS and Burnett JC Jr: Brain natriuretic peptide enhances renal actions of furosemide and suppresses furosemide-induced aldosterone activation in experimental heart failure. Circulation 109: 1680-1685, 2004.

22. Durak-Nalbantić A, Džubur A, Dilić M, Pozderac Z, Mujanović-Narančić A, Kulić M, Hodžić E, Resić N, Brdjanović S and Zvizdić F: Brain natriuretic peptide release in acute myocardial infarction. Bosn J Basic Med Sci 12: 164-168, 2012.

23. Eindhoven JA, van den Bosch AE, Jansen PR, Boersma E and Roos-Hesselink JW: The usefulness of brain natriuretic peptide in complex congenital heart disease: A systematic review. J Am Coll Cardiol 60: 2140-2149, 2012. 
24. Roberts E, Ludman AJ, Dworzynski K, Al-Mohammad A, Cowie MR, McMurray JJ and Mant J; NICE Guideline Development Group for Acute Heart Failure: The diagnostic accuracy of the natriuretic peptides in heart failure: Systematic review and diagnostic meta-analysis in the acute care setting. BMJ 350: h910, 2015.

25. Di Angelantonio E, Chowdhury R, Sarwar N, Ray KK, Gobin R, Saleheen D, Thompson A, Gudnason V, Sattar N and Danesh J: B-type natriuretic peptides and cardiovascular risk: Systematic review and meta-analysis of 40 prospective studies. Circulation 120: 2177-2187, 2009.

26. van Veldhuisen DJ, Linssen GC, Jaarsma T, van Gilst WH, Hoes AW, Tijssen JG, Paulus WJ, Voors AA and Hillege HL: $\mathrm{B}$-type natriuretic peptide and prognosis in heart failure patients with preserved and reduced ejection fraction. J Am Coll Cardiol 61: 1498-1506, 2013.
27. Iacoviello M, Leone M, Antoncecchi V and Ciccone MM: Evaluation of chronic kidney disease in chronic heart failure: From biomarkers to arterial renal resistances. World J Clin Cases 3: 10-19, 2015.

28. Koglin J, Pehlivanli S, Schwaiblmair M, Vogeser M, Cremer P and vonScheidt W: Role of brain natriuretic peptide in risk stratification of patients with congestive heart failure. J Am Coll Cardiol 38: 1934-1941, 2001

29. Shlipak MG, Chertow GC and Massie BM: Beware the rising creatinine level. J Card Fail 9: 26-28, 2003.

30. Arakawa K, Ando Y, Matsunaga S and Nishiura A: Diagnostic ability of natriuretic peptides for heart failure according to eGFR level: Comparison between brain natriuretic peptide and amino-terminal probrain natriuretic peptide. Rinsho Byori 64: 251-257, 2016 (In Japanese). 